\title{
Diffusion effect analysis of pollution gas under the impact of urban three-dimensional pattern
}

\author{
Li Sui ${ }^{1, a}$, Jiang Mingzhuo ${ }^{1, b}$, Li Zhenxing ${ }^{1, c}$ and Zhou Shiwen ${ }^{1, d}$ \\ ${ }^{1}$ Ecological Urbanization and Green Construction Research Center, Shenyang Jianzhu University, \\ Shenyang 110168

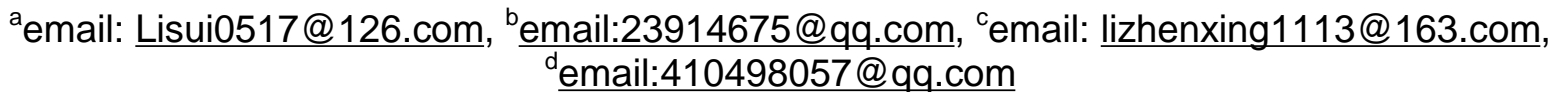

Keywords: three-dimensional landscape pattern; air pollutant; commercial district; numerical simulation; diffusion effects

Abstract: The paper applies CFD numerical simulation technology, selects the typical urban commercial block as the research object, uses PM10 which are from automobile exhausted gas as the source of pollution, simulates the spatial density distribution of the inhalable particle, verifies the accuracy based on the monitoring data and reveals the diffusion rule of atmospheric pollutants in the three dimensional block space. The results show that there is a close relationship between the diffusion effect of the contaminated gas and three dimensional landscape pattern in blocks. At different height levels such as 10 meters, 30 meters and 50 meters, the decreasing trend of the density of the pollutants and the uniformity of the spatial distribution are quite obvious. The height of the buildings along the streets, the space combination and the shape of the buildings themselves determine the inner smoothness of the streets. In the urban planning, one must fully consider the effect of three dimensional pattern on air pollution in blocks. From the aspects of urban ventilation, open space, height of the horizon line and disheartened space of the bottom, three dimensional landscape pattern of urban blocks should be optimized, which can effectively improve the current situation of severe atmospheric pollution.

Three-dimensional landscape pattern of urban blocks is an important factor determining air flow and diffusion effect of atmospheric pollutants within the blocks. It also affects the quality of internal atmospheric environment ${ }^{[1]}$. The spatial layout structure of urban high-density commercial district is complex with rich combination of the building blocks and diverse forms of the buildings themselves. With the joint action of the urban meteorological elements, the micro climate of an urban area is formed ${ }^{[2-3]}$. The paper undertakes CFD numerical simulation of air movement at different spaces and diffusion of inhalable particle PM10 within typical urban business districts, analyzes the impact mechanisms of three-dimensional pattern on the diffusion effects of polluting gases, and puts forward different optimization schemes of spatial patterns on the basis of the results. Moreover, it provides a theoretical basis for the reasonable layout of urban block space.

\section{Research area and research methods}

\section{Research area}

The research selects Shenyang's Taiyuan Street as the research object. The research scope ranges east to Nanjing Street, close to Zhongshan Park, west to Shengli Street and Shenyang Station, north to Beier Road, south to Nanwu Road. It takes vehicle emissions of PM10 at three main roads, namely Zhonghua Road, Zhongshan Road and Minzhu Road as the source of pollution. The region has the following characteristics: (1) It is adjacent to Shenyang Station and is the transportation hub with heavy traffic. The pollution of atmospheric particles is more serious; (2) It is Shenyang's core business economic district. People's outdoor activities at commercial pedestrians are quite active and the atmospheric micro-environment needs to be improved; (3) The block's three-dimensional landscape pattern has the characteristics of a high density urban business district with high-density building blocks and diverse forms of buildings' layout ; (4) The structure of the underlying surface 
is complex with few green trees and dense road network. The streets stretch to different directions and is not prone to the spread of pollutants.

\section{Data sources}

The numerical value of PM10 quality density coming from road vehicle emissions at Taiyuan Street are monitored by CPR-KA type air quality monitor. At different traffic times in October--November, 2015, several field monitoring are undertaken. Meteorological elements, including daily average wind speed and relative humidity are derived from the annual statistics of Environmental Monitoring Experiment Center, Liaoning Province. Origin software is applied to process the quality and density data of PM10. Matlab software is applied to remove the inhalable particles and meteorological anomalies in the data. All these serve as the basis for setting the boundary conditions in the CFD model.

\section{Research methods}

The research applies the method of monitor - simulation - verification and establishes a three-dimensional spatial numerical model based on CFD. The distribution of particle density under the three-dimensional pattern in blocks is stimulated in accordance with the quality and density of the measured street particulate emissions as well as the set boundary conditions of meteorological factors. Moreover, field monitoring data is applied for verifying the model accuracy, which reveals the impact of the three-dimensional spatial pattern of particle diffusion ${ }^{[4]}$.

During the numerical simulation process, the release speed of pollutants is determined according to the traffic flow and the speed of the emissions. This study sets the pollution source to one meter liner pollution source on the supposition that the emission manner and the density is stable. The density of the pollutants is $0.08 \mathrm{mg} / \mathrm{m}^{3}$ at a speed of $0.5 \mathrm{~m} / \mathrm{s}$ with a vertically upwards direction ${ }^{[5]}$.

\section{Establishment of three-dimensional model}

In the research area, the main roads, namely Zhonghua Road is about 910m, Zhongshan Road is about $510 \mathrm{~m}$ and Minzhu Road is about $580 \mathrm{~m}$. Through field reconnaissance, three-dimensional pattern is established with the use of Gambit based on the external shape of the original buildings. According to the related research, in order to ensure the full development of traffic, the computational area of simulation experience is a cabinet space of $1800 \mathrm{~m} * 1800 \mathrm{~m} * 300 \mathrm{~m}$.

\section{Meshing generation and set of boundary conditions}

To improve the accuracy of the simulated test, one applies unstructured mesh generation in Gambit and undertakes mesh refinement near the ground and inside the street, forming an analysis grid ranging from $10 \mathrm{~m} \sim 20 \mathrm{~m}$. The annual average wind speed of Shenyang is $3 \mathrm{~m} / \mathrm{s}$ in the north in winter, so the inflow wind speed is set to be $3 \mathrm{~m} / \mathrm{s}$ and the turbulence intensity is set to be $2 \%$. The wind comes from the north. The exit of the atmosphere is the boundary condition for full development. The upper boundary is a given symmetry boundary. The internal interface within the block uses no-slip condition. Walls near uses wall-function method to process ${ }^{[6]}$.

\section{Select of control equation}

The block model established by gambit is led to a simulation software called fluent to set the relevant hydrodynamic conditions and at least 500 steps of iteration. During the simulation process, the equations applied is ${ }^{[7-12]}$ :

(1) continuity equation

$$
\frac{\partial \bar{U}_{i}}{\partial x_{i}}=0 \quad i=1,2
$$

(2) conservation of momentum equation

$$
\frac{\partial \bar{U}_{i}}{\partial t}+\bar{U}_{j} \frac{\partial \bar{U}_{i}}{\partial x_{j}}=-\frac{1}{\rho} \frac{\partial \bar{p}}{\partial x_{i}}-\frac{\partial}{\partial x_{j}}\left(\overline{u_{i} u_{j}}\right)+v \nabla^{2} \bar{U} \quad j=1,2
$$


$\bar{p}-: \quad$ average time the fluid pressure

$\rho-$ fuid density

$v$ - fluid kinematic viscosity coefficient

$u_{i}, u_{j}-$ pulsating flow velocity in the $i$ and $i$ components

$\overline{u_{i} u_{j}}$ - pulsating fluid tangential stress

$x_{i}, x_{j}-$ coordinates $i$ and $i$ directions

$\bar{U}_{i}, \bar{U}_{j}$ - fluid velocity in both directions on $\mathrm{i}$ and $\mathrm{j}$ are the

(Standard equations $k-\varepsilon$ )

$$
\begin{gathered}
\frac{\partial k}{\partial t}+\bar{U}_{j} \frac{\partial k}{\partial x_{j}}=\frac{\partial}{\partial x_{j}}\left(\frac{v_{t}}{\sigma_{k}} \frac{\partial k}{\partial x_{j}}\right)+v_{t}\left(\frac{\partial \bar{U}_{i}}{\partial x_{j}}+\frac{\partial \bar{U}_{j}}{\partial x_{i}}\right) \frac{\partial \bar{U}_{i}}{\partial x_{j}}-\varepsilon \\
\frac{\partial \varepsilon}{\partial t}+\bar{U}_{j} \frac{\partial \varepsilon}{\partial x_{j}}=\frac{\partial}{\partial x_{j}}\left(\frac{v_{t}}{\sigma_{\varepsilon}} \frac{\partial \varepsilon}{\partial x_{j}}\right)+\frac{\varepsilon}{k}\left[C_{\varepsilon 1} v\left(\frac{\partial \bar{U}_{i}}{\partial x_{j}}+\frac{\partial \bar{U}_{j}}{\partial x_{i}}\right) \frac{\partial \bar{U}_{i}}{\partial x_{j}}-C_{\varepsilon 2} \varepsilon\right] \\
\varepsilon-\text { Turbulence dissipation rate }
\end{gathered}
$$

(3) Component equation

$$
\begin{gathered}
\frac{\partial\left(\rho c_{s}\right)}{\partial t}+\operatorname{div}\left(\rho u c_{s}\right)=\operatorname{div}\left(D_{s} \operatorname{grad}\left(\rho c_{s}\right)\right)+s_{s} \\
c_{s}-\text { volume concentration of component s } \\
\rho c_{s}-\text { concentration of component s } \\
D_{s}-\text { diffusion coefficient of component s }
\end{gathered}
$$

\section{Verification of simulation results}

In this simulation, the verification data mainly applies a variety of monitoring data and the data verification points mainly distribute at streets and commercial areas within Taiyuan Street in Shenyang. 15 monitoring points are selected to verify the results of model simulations. The results show that it is feasible to use Fluent to stimulate the diffusion and distribution of PM10.

\section{Results and analysis}

\section{distribution rule of pollutants at the horizontal section of block X-Y Select of typical horizontal section}

The three-dimensional block pattern changes obviously as the height increases. The building density gradually decreases at different heights, and three streets turn out $90^{\circ}, 45^{\circ}, 0^{\circ}$ angle with the dominant wind direction. Figure 2 is the particle density distribution at heights of $1.5 \mathrm{~m}, 10 \mathrm{~m}$, $30 \mathrm{~m}, 50 \mathrm{~m}, 80 \mathrm{~m}$ in different horizontal section. It shows that the distribution uniformity degree and the decreasing trend density of atmospheric pollutants are different at different horizons. 

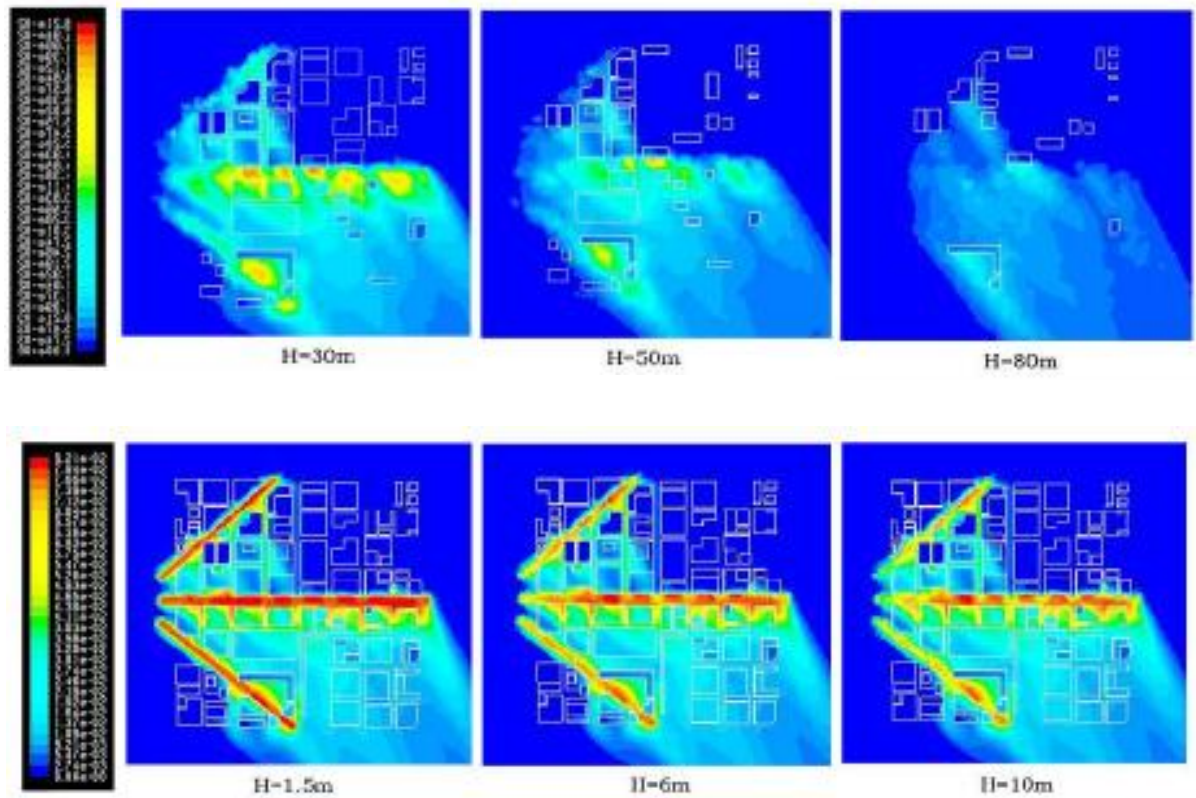

Fig. 1 Pollutant diffusion distribution at X-Y horizontal section of different heights

Effect analysis of three-dimensional pattern to pollutants' density distribution at different horizons

Table 1 is the description and cause analysis of the simulation results. It reveals the different building density, the street direction, the interface continuity of streets and the distribution characteristics of air pollutants at open space under the X-Y coordinates. Thus the diffusion effect of above factors on pollution gas can be compared.

\section{Distribution rule of Pollutants ate cross-section of block $\mathrm{Y}-\mathrm{Z}$ coordinates}

\section{Select of the cross-section}

Select different cross-sections at Y-Z coordinate and investigate the effect of street canyon geometry and construction form on the diffusion of atmosphere pollutants according to the simulation results. Zhonghua Road has a total width $\mathrm{W}=40 \mathrm{~m}$ and the width of the liner sources is $\mathrm{W} 1=20 \mathrm{~m}$, which is also called road red line. The width of the square between the commercial buildings on both sides and the red line is between $\mathrm{W} 2=\mathrm{W} 3=10 \mathrm{~m}$. On both sides of the street are the buildings upstream and downstream of the wind. $\mathrm{H} 1$ and $\mathrm{H} 2$ ares the height of the buildings on both sides. Select eight typical sections, section details are shown in Table 3.

\section{Analysis of pollutants' diffusion effect at different geometry cross-section}

As shown in Figure 2-a(section 1-1), H1: H2: W $=1.25: 1$ : 1, it is visible that the density of pollutants within the block is much higher with larger polluted areas. As the ratio of height and width increases, the air flow is more difficult to enter the street canyons. Since the upwind buildings are higher than the downwind buildings, the "climb the wall effect" becomes more obvious.

As shown in Figure 2-b (2-2 section), H1: H2: W = 4: 1: 2. The upwind buildings are significantly higher than the downstream buildings. The downwind forms a large-scale vortex. The vortex center moves upward to the top of the downwind buildings. The "climb the wall effect" makes the pollutants form a large contaminated area on the leeward side of high-rise buildings. This shows that the increase of the upwind buildings' height may cause pollutants climb to the vertical direction of the leeside, increasing the scope of pollution, which is extremely conducive to the spread of pollutants within the street canyons. 


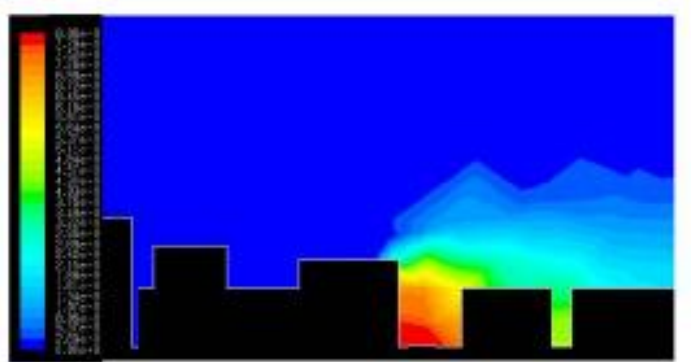

$1-1$

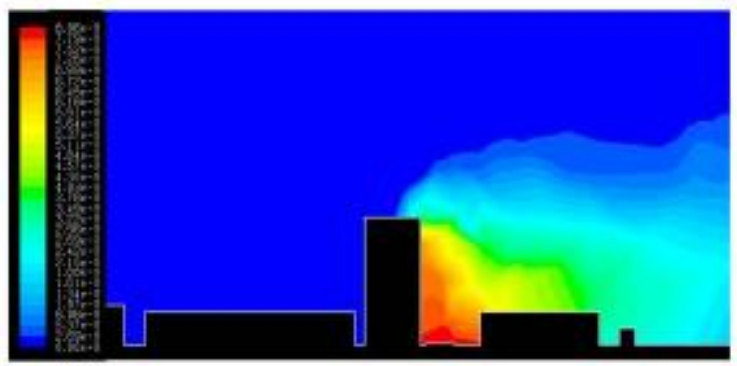

$2-2$

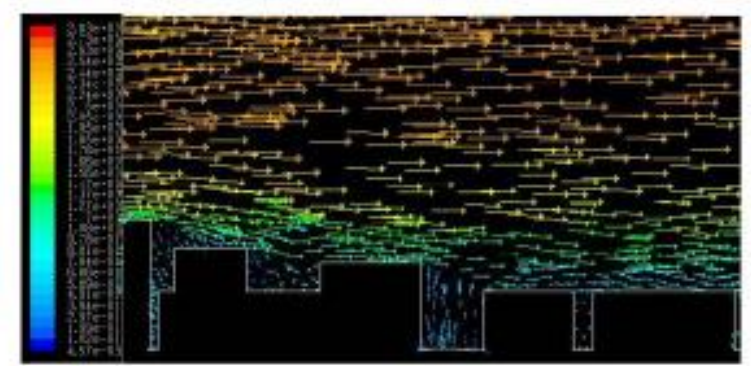

$1-1$

2-a

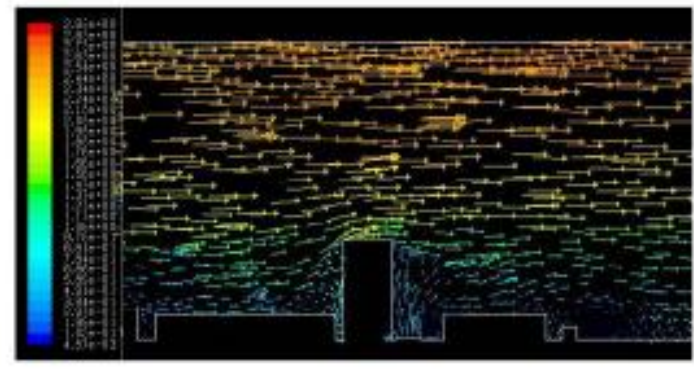

2-b

$2-2$

\section{Discussion}

The micro-environmental differences under different three-dimensional patterns at urban blocks is significant, particularly the complex form of the space. The urban commercial districts have diverse building forms of their own. Therefore, one applies CFD numerical simulation technology in urban design and optimize the three-dimensional landscape pattern based on the simulation results, which is finding a way to deal with air pollutants. In this study, analysis is made based on the simulation of inhalable particles' diffusion at Taiyuan Street and recommendations is put forward for optimizing the layout of the building space:

(1) Ventilation corridor design: At a high-density urban space, ventilation corridor setting is conducive to promoting the healthy functioning of local flow. When the ventilation corridor conforms to the prevailing wind of the city, the angle of the corridor and the wind is between $30^{\circ}$ $-60^{\circ}$. When the main exit corridor eventually conforms to the prevailing wind direction, the corridor has good effect of ventilation. After entering the corridor, the upstream wind will change with the form of the corridor and enter the secondary corridors which are connected with the main corridor. In urban design, one should fully consider the main functions of urban streets, landscape ecological linear corridors and other open space to cities' ventilation. The construction of ventilation corridor based on CFD technology helps enhance the ventilation effect.

(2) Integration of open space: The density of urban commercial districts is large with generally less open space. During the process of simulation and analysis, we found the density of pollutants at a large open space is significantly reduced. Therefore, combining urban functions to design some open space is an important way to improve the commercial districts' pollution. The use of CFD techniques can simulate regional wind flow in order to determine the layout of public open space set open space suitable for different seasons depending on the wind characteristic in summer and winter at different places .

(3) Skyline control : Change of the height of the buildings can lead to the change of partial wind flow. This phenomenon is the most evident in an open area or streets with distinct differences of buildings' height on both sides. For the urban commercial districts, at the height of 50-100 meters, the spatial distribution of high-rise towers has a significant impact on the bottom flow field of street canyons. In urban design, skyline control should have an overall consideration with rich transition level elevation to avoid worse local pollution caused by sudden altitude changes. 


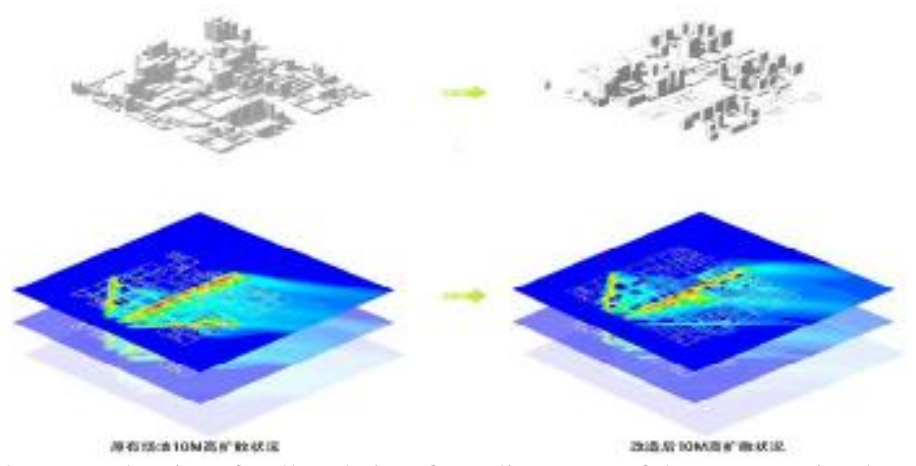

Figure 3. Simulation comparison between density of polluted airs after adjustment of the construction layout

\section{Conclusions}

The paper takes Shenyang's climatic conditions as the basis for test simulation. The research object Taiyuan Street has the typical characteristics of the commercial districts. The analysis conclusion applies to other urban blocks with the same climatic conditions like Shenyang. Through simulating the diffusion effects of pollutants at horizontal and vertical space, it turns out that the density space distribution of inhalable particles has a close relationship with three-dimensional patterns.

1) At respective horizontal cross-sections at the height of 10 meters below, the density of the buildings substantially has no difference. Except at the various points of the streets outside pollution sources, the distribution of particles is even with no obvious change of density. To the height of 30 $\mathrm{m}$, buildings' density decreases and the density of pollutants turns out an obvious decreasing trend. In the range of 50-80 meters, the density of buildings decreases dramatically and the pollutants basically have all spread.

2) At the horizontal section of the block, due to the difference of buildings' spatial combination and buildings forms of their own, the change trend of pollutants' density is quite different at different typical spaces in the vertical direction different. At the height ranging from $10 \mathrm{~m}-30 \mathrm{~m}$, the density of each point at the space has a big difference. The distribution of pollutants within the inner space with buildings which are different along the street canyons is very uneven. Most pollutants gathered on the leeward side of the upstream buildings.

3) At the cross-section of the block, when the ratio of the buildings' height along the street canyons and the street's width is $\mathrm{H} 1: \mathrm{H} 2: \mathrm{W}=1: 1: 4$, the space is open and the wind can fully enter the street canyons which is conductive to dirty gas dilution. When $\mathrm{H} 1: \mathrm{H} 2: \mathrm{W}=1: 1: 2$, only part of the wind goes into the bottom through the down wash and the swirl takes away some pollutants. Upstream buildings is lower than downstream buildings, which is more conducive to the spread of pollutants.

The above analysis shows, the three-dimensional pattern of blocks and the density field of atmospheric pollutants have a strong correlation, thus forming a rational spatial pattern through urban design and can effectively increase the climate adaptation of street space, enhance the quality of the atmospheric environment. At the same time, the air purification function of the ecological infrastructure should be made full use. Moreover, one should combine airy corridors with open space design, make full use of the green system for releasing oxygen and decreasing dust. Furthermore, one should strengthen the adaptability of the combination of urban space and form to urban wind field. The research applies the simulation techniques to reveal the distribution rule of pollutants, improves the grim status quo of air pollution through urban planning and design, which has important guiding significance for the optimization of an three-dimensional landscape pattern .

\section{References}

[1] Gong Jiping, Hu Yuanman, Liu Miao, Chang Yu, Bu Rencang, Xiong Zaiping (, Li Chunlin. 
Three Dimensional Expansion of Urban Landscape and its Effect on Atmospheric Environment. Chinese Journal of Ecology, , 2015,34(2): 562-570 (in Chinese)

[2] M.A.Salam, Y.Shirasuna, K.Hirano, et al. Particle Associated Polycyclic Aromatic Hydrocarbons in the Atmospheric Environment of Urban and Suburban Residential Area. International Journal of Environmental Science \& Technology, 2011, 8 (2): 255-266

[3] Zou Yuan, Hu Chunsheng, The Influence Factors of Urban Micro Climate and Regional Information Map. Journal of Wuhan University of Technology,2008,30(8): 178-180+196(in Chinese)

[4] Li Shui, Zhu Lei, Shi Tiemao, Wang Wei. Planning Strategies for the Prevention and Control of Particulate Pollution in Urban Blocks. Urban Development Studies,2014,21(1): 42-45(in Chinese)

[5] Wang Jiwu, Mu Yin.Study on Spatial Strategy Responding to Automobile Exhaust Pollution in Urban Street Canyons.City Planning Review ,2013,37(5): 54-60 (in Chinese)

[6] Zhao Jingyuan, Liu Jiaping. Digital Simulation of Thermal Environment and Countermeasures against the Planning and Design for Urban Street Canyon. Architectural Journal,2007, (3): 37-39(in Chinese)

[7] Yang Li. The Application of CFD Technology in the Analysis of Wind Environment in Residential Area. Architectural Journal,2010, (S1): 5-9(in Chinese)

[8] Zhao Jingping, Liu Jiaping. Dynamic Thermal Effect of Urban Street Landscape. Energiae Solaris Sinica,2009,30(8): 1013-1017(in Chinese)

[9] Song Xuan, Duan Jinlong, Du Liping. The research on Urban Heat Island Effect. Meteorological and Environmental Sciences,2009, (03): 68-72(in Chinese)

[10] Wu Zhenzhen, Yan Tao,Fu Xezhao.CFD Simulation Technology based Analysis on Urban Wind Environment of Shenzhen. Construction Quality,2009,27(11): 49-53(in Chinese)

[11] Zheng Shuanning,Su Xiaodan,Wang Haowei, Li Chunming, Wang Cuiping, Dong Rencai. Research Progress of Natural Ventilation in Urban Environment. Environmental Science \& Technology,2012,35(4): 87-93(in Chinese)

[12] Li Siu, Zhu Lei, Shi Tiemao, Liu Min, Yang Erdong. Study on the Planning Design for Coastal Community Based on Simulation and Optimization of the Wind Environment. Journal of Shenyang Jianzhu University(Natural Science), ,2015,31(1): 173-181(in Chinese) 